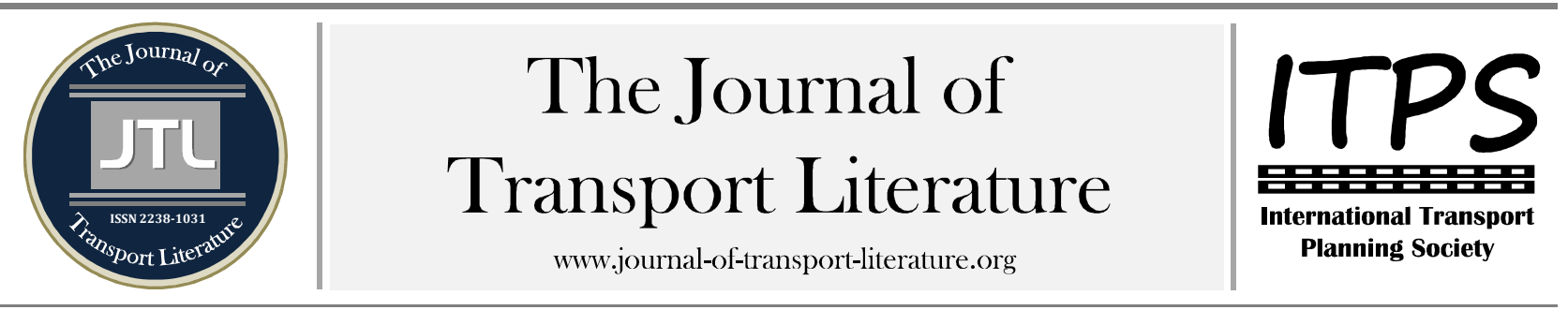

\title{
Developing corridor choice model under hypothetical Variable Message Sign based traffic information
}

\author{
Debasis Basu1,+; Bhargab Maitra ${ }^{2}$ \\ 1 Indian Institute of Technology Bhubaneswar, Bhubaneswar, India \\ 2 Indian Institute of Technology Kharagpur, Kharagpur, India
}

\section{Article Info}

Keywords:

corridor/route choice

revealed preference Stated

preference

logit model

variable message sign

\section{Abstract}

The article presents development of a closed-form corridor choice model under hypothetical variable message sign (VMS) based traffic information. A single VMS board is assumed to display traffic information at a junction of two alternative and competitive traffic corridors connecting two catchment areas in Kolkata city, India. The corridor choice models are developed by combining revealed preference (RP) and stated preference (SP) data sources. The development of a combined RP-SP model is a challenging task as different data sources have their respective error terms. In this work, the data sources are combined by exploiting their respective merits; while discarding their respective de-merits. Here a procedure of developing composite utility function is presented, which is constituted of estimates of attributes from SP data source and alternative specific constant term of alternatives calibrated from RP data source, while fixing all coefficients of attributes at SP estimates. The construction of corridor choice models is demonstrated for two types of VMS based traffic information, which differs in terms of the content displayed on VMS board and also for two types of trip maker- namely private car and taxi. Under the influence of VMS-based traffic information, trip makers are found to take corridor choice decision based on the rational trade-off between travel time information and direct travel cost of alternative traffic corridors. The alternative-specific-constant term of choice models indicates that in presence of VMSbased information, private car trip makers are likely to be less biased to choose comparatively longer but almost free-flow traffic corridor (thereby less travel time corridor); while taxi trip makers are likely to be more biased to choose longer but almost free-flow traffic corridor to arrive at their destination.
Submitted 25 Mar 2014; received in revised form 3 Sep 2014 accepted 8 Oct 2014.

Licensed under

Creative Common

CC-BY $3.0 \mathrm{BR}$

+ Corresponding author. School of Infrastructure. Indian Institute of Technology Bhubaneswar. Samantapuri, Bhubaneswar - 751 013. India.

E-mail address: basudebasis2k@gmail.com, dbasu@iitbbs.ac.in

\section{Introduction}

Route (or say corridor) choice model is essential for forecasting traffic demand on alternative traffic routes in an urban area. It evaluates travel cost of all alternative routes from an origin to a destination and helps to estimate route switching behavior under various hypothetical travel scenarios and thereby, it facilitates to analyze likely traffic condition on the transportation network. The article presents a procedure of the development of route choice model of trip makers under hypothetical under variable message sign (VMS) based traffic information travel scenarios.

VMS is a programmable traffic guidance device, which displays non-personalized real-time information of traffic on alternative routes/corridors to trip makers and drivers. In recent years, there has been a growing trend of providing real time traffic information through VMS board as an instrument for temporal and spatial management of traffic congestion (Peeta et al. 2000; Chaterjee et al. 2002; Baofeng et al. 2005; Bierlaire et al. 2006; Basu and Maitra, 2007 and 2010a). VMS is proven to be cost-effective measure (Adler and Blue 1998; Peeta and Ramos 2006) for mitigating traffic congestion. Many previous investigations showed that, if real time traffic information via roadside VMS was provided to trip makers in some strategically selected locations, then a change in route switching behavior among trip makers and drivers was expected. It is believed that traffic information helps to overcome the behavioral inertia and because of that individuals are more likely to change their travel patterns in response to information (Khattak et al. 1996). The VMS is expected to bring more benefit to the traffic stream, when the operating traffic volume approaches to capacity. In this condition, a small reduction in traffic volume due to a change in route choice behavior under the influence of VMS is likely to bring significant benefit to trip makers (Basu and Maitra, 2010b). The effect of VMS based traffic information on trip makers greatly depends on the content and format (Chatterjee et al. 2002; Peeta et al. 2000) of VMS based information.

Various researchers studied in-laboratory the effect of VMS-based traffic information on traffic stream. The University of Leeds employed VLADIMIR route-choice simulator (Bonsall et al. 1997) to investigate drivers' response to VMS. Richards et al. (1996) used the RGCONTRAM model to study factors influencing the effectiveness of VMS. RGCONTRAM was used to analyze the impact of VMS on motorways (McDonald and Richards, 1996). Some reserchers (Lim and Lee, 1997; Lam and Chan 2001; Basu and Maitra, 2010b) developed dynamic traffic assignment (DTA) model to investigate the same. One of the key inputs for such in-laboratory based evaluation of VMS-based policies in a dynamic-paradigm is route choice model of 
trip makers and drivers under the influence of VMS-based traffic information. The success of in-laboratory based evaluation of VMS lies to a great extent on accurate forecasting of traffic on alternative routes by route choice model. In this case, many researchers proposed the use of combining two different data sources such as revealed preference (RP) and stated preference (SP). The development of a combined RP-SP model is a challenging task as different data sources have their respective error terms (Ben-Akiva and Morikawa, 1990; Bradley and Daly, 1994). The objective of the present article is to develop a closed-form logit-based corridor choice model by combining RP and SP data sources. The data sources are combined by exploiting their respective merits; while discarding their respective de-merits. The work is demonstrated by taking a case study in Kolkata city, where a single VMS board is assumed to display traffic information at a junction of two alternative and competitive traffic corridors connecting two catchment areas. It considers two types of hypothetical VMS based traffic information, which differs in terms of the content displayed on VMS board and assumes that all trip makers are expected to comply with traffic information and they choose corridor based on their lowest perceived travel cost. The presentation of this article is carried under following sections: 1 . Approach and methodology; 2 Procedure of developing composite utility function, 3 Empirical study and then Conclusions.

\section{Approach and Methodology}

Several researchers (Peeta et al. 2000; Basu and Maitra, 2007 and 2010a) investigated the effect of hypothetical VMS based traffic information on trip makers using stated preference (SP) data. SP data primarily represents respondents' preference (say choice) under given hypothetical condition, and so it is unable to simulate actual market condition. Therefore, the estimated coefficients of alternative-specific-constant from SP data have no behavioral meaning, especially for the investigation relating to demand forecast unless a care is taken on calibration of the alternative specific constant term of utility function. In discrete choice model, alternative-specific-constant term represents choice shares of respective alternative in sample data set (Train, 2003; Hensher et al. 2005). On the other hand, by nature RP data is found almost as invariant in terms of attributes and its levels, (Louviere et al. 2000; Hensher et al. 2005). Therefore, coefficient estimate of alternative-specific-constant using RP data indicates biasness of individuals' choice preference to a particular alternative. The above fact is highlighted in established literature by many researchers and therefore, researchers suggest combination of RP and SP data sources. The process of combining of these data sources is called data-enrichment (Louviere et al. 2000). Later, many researchers (Ben-Akiva and Morikawa, 1990; Bradley and Daly, 1994; Hensher and Bradley, 1993; Hensher, 1998 and so on) also have addressed the data-enrichment process in many ways. The major purpose of this aspect of research is to develop a model, which can more accurately forecast the demand.

The problem associated with the combination of two different data sources is the type of their error terms. In case of RP data, errors are due to attributes; whereas in case of SP data errors are due to the types of choice made by an individual during SP survey. In the present study, data enrichment is carried out using a procedure described by Hensher et al. (2005) through construction of composite utility function using RP and SP data sources, which is adopted in the present study.

\section{Procedure of Developing Composite Utility Function}

In the present work, the Multinomial Logit (MNL) (Mc-Fadden, 1974) model is developed using maximum likelihood technique. Initially, the model is developed for RP and SP datasets. Later, the combined model of RP and SP data sources has been developed through the construction of a composite utility function using a procedure described in Hensher et al. 2005 by calibration of alternative specific constant term. In this procedure, the composite utility function is developed by discarding the RP attribute estimates and the SP constant terms, but by keeping the SP attribute estimates and the alternative specific constant terms recalibrated from RP data; while estimating coefficients of attributes using SP data. The process of re-calibration of alternative specific term is described below. Say, the systematic (i.e. representative) utility function of an alternative $\mathrm{i}$ is described by k number of attributes (such as $\mathrm{x} 1, \mathrm{x} 2, \ldots, \mathrm{xk}$ ). Then, the systematic utility function becomes

$$
V_{i}=\beta_{0 i}+\beta_{1} x_{i 1}+\beta_{2} x_{i 2}+\cdots+\beta_{k} x_{i k}
$$

Where, $\beta 0 \mathrm{i}$ is an alternative specific constant term for alternative i. It can be calibrated using the following formula by either of RP or SP data source.

$$
\beta_{0 \mathrm{i}}=\overline{\mathrm{V}}_{\mathrm{i}}-\sum_{\mathrm{k}=1}^{\mathrm{K}} \beta_{\mathrm{k}} \mathrm{x}_{\mathrm{k}}
$$

Here, in the proposed procedure the alternative specific constant term is recalibrated from RP data source, where the later part of the above equation considers the SP data source.

Therefore, the above equation can be re-written in the following way

$$
\beta_{0 \mathrm{i}}^{\mathrm{RP}}=\overline{\mathrm{V}}_{\mathrm{i}}^{\mathrm{RP}}-\sum_{\mathrm{k}=1}^{\mathrm{K}} \lambda^{\mathrm{SP}} \beta_{\mathrm{k}}^{\mathrm{SP}} \mathrm{x}_{\mathrm{k}}^{\mathrm{SP}}
$$

Where $\lambda \mathrm{SP}$ is called the scale parameter of SP data, which is inversely proportional to its variance. Therefore, the composite utility function (say, $V_{\text {Com(i) }}$ ) of alternative i for the combined RP and SP data sources can be constructed as per the following

$$
\mathrm{V}_{\text {ComUtility (i) }}=\beta_{0 i}^{R P}+\sum_{k=1}^{K} \lambda^{S P} \beta_{k}^{S P} x_{k}^{S P}
$$

\section{Empirical Study}

\subsection{Study Area, Attributes and Survey}

Two, alternative and competitive to each other traffic corridors are selected in the Kolkata city, India, which provide oneway traffic movement service between two major catchment areas, namely Park Circus and Esplanade/Dalhousie area of CBD (Central Business District), Kolkata. The study corridors (called as fly-over (FO) corridor and Park Street (PS) corridor) 
majorly carry private cars and taxies. The length of the FO corridor is $5 \mathrm{~km}$.; whereas the length of the PS corridor is $3.4 \mathrm{~km}$. The journey time along FO corridor varies between 7 to 9 minutes; whereas along PS it varies between 20 to 28 minutes. A single VMS is assumed to display traffic information at the junction of the two traffic corridors. The current work develops choice model for two types of trip maker namely private cars and taxies as they are prevalent on these corridors. In this work the empirical model of demand forecasting for each type of trip maker is also seperately attempted for two types of VMS based traffic information, which differs in terms of the content displayed on VMS board. One, the instantaneous travel time alone (called as TI-I) and the other, the instantaneous travel time along with its variation level (called as TI-II). Here four types of variation level such as low, medium, high and very high are considered. The work assumes that trip makers perceive the VMS based traffic information and choose corridor based on their lowest perceived travel dis-utility. A choice based SP survey was conducted for two types of traffic information and also for two types of trip maker in order to observe trip makers' corridor choice behavior under various hypothetical scenarios of traffic information. A paper-pencil based survey was carried out in the month of May-June, 2005. A total of 3651 and 3857 SP observations of traffic information TI-I are obtained for private car and taxi trip makers respectively. Similarly, a total of 4643 and 5053 observations of traffic information TI-II are obtained for private car and taxi trip makers respectively.

\subsection{Model development}

In this work, travel time and direct travel cost (say fuel consumption in case of private cars and fare for taxi users) are found to be most important attributes of concern to a trip maker (He et al. 2003; Basu and Maitra, 2010b). Therefore, this study primarily considers these two attributes. In all models, alternative specific constant term for flyover (FO) corridor is estimated with respect to Park Street (PS) corridor. Travel time and travel cost are considered as independent attributes in the utility model specification for TI-I. For TI-II, variation level of traffic information is considered in addition to travel time and travel cost attribute. For both TI-I and TI-II, data for travel time and travel cost are entered in cardinal linear (i.e. continuous scale) form. In order to capture non-linear effect of the variation level of TI-II on traffic stream, effects-coding ($1,0,+1)$ technique is employed considering the low variation level as the base level. Initially, MNL model of corridor choice (over-lapping of corridors is absence) is developed for private and taxi trip makers from RP and SP data sources. Then, recalibration of alternative specific constant term for FO corridor is done as per the procedure described in section 2 . In the present study, SP data set of TI-II has unique attribute called as variation level of travel time information, which does not exists in the RP counterpart. Therefore, during recalibration of the alternative specific constant term for TI-II, the coefficient estimates of different variation-level emanating from SP data source is kept out of the estimation process. But they are finally incorporated during construction of composite utility function, which is used for demand forecasting under TI-II.

\subsection{Results and Discussion}

The MNL models for private car and taxi trip makers from RP data set, SP data set and then re-calibration of alternative specific constant term for FO corridor are reported in Table 1 and Table 2 for TI-I and TI-II respectively. Overall, coefficients of all attributes are estimated with significant t-statistics (i.e.more than 1.96), indicating that the estimated coefficients are significantly (more than 95\% confidence level) different from zero. In this aspect it is required to mention that during initial model run, alternative specific constant term for private car trip makers was estimated with insignificant t-statistics for SP model under TI-II. Therefore, the SP model is re-estimated (as reported here in Table 2) without considering the alternative specific constant term. The sign of coefficient is also observed, which is in agreement with the case study. Sign of coefficients of travel time and travel cost attribute is found to be negative representing these attributes as dis-utility; whereas the change of sign of coefficients of variation level of traffic information from medium to high or very high is found to be changing from positive to negative, indicating higher variation levels as dis-utility with respect to lower variation level. The sign of alternative specific constant term for FO corridor is found negative, indicating trip makers are not biased towards choosing of FO corridor. The authors carried out a validation study of the RP model in a dynamic paradigm. A dynamic traffic assignment (DTA) model is developed under no-traffic-information scenario (which is not the scope of present study). The validation study revealed that the modal split of private cars and taxies on alternative study corridors was found almost similar with that of actual observation. Besides, the model estimated travel time from Park circus to Esplanade area is found to vary between 5-10 minutes on FO corridor and 20-30 minutes on PS corridor. This result is almost in agreement with the observed travel time on the respective study corridors. A comparison of the estimated coefficients of alternativespecific-constant term between hypothetical scenario (i.e. traffic information TI-I and TI-II) and no-traffic-information scenario (i.e. the current traffic movement pattern) is carried out for private car and taxi trip makers. In this regard, the alternative specific constant term re-calibrated from combined RP and SP data sources (i.e. traffic information scenarios) is compared with that of RP data source (i.e. no-traffic-information).

For private car trip makers, it is observed (Table 1 and 2) that estimated value of alternative specific constant under traffic information scenario is less than that of no-traffic-information scenario. This indicates that private car trip makers' corridor choice behavior is comparatively less biased towards FO corridor under traffic information. This may be justified by the fact that under a given traffic information scenario private car trip makers get less biased to choose longer FO corridor in order to reduce their direct travel cost for a given budget of travel time. Under traffic information scenario, private car trip makers are expected to take decision based on rational trade-off between travel time and direct travel cost of alternative study corridors. A similar type of comparison is also carried out for taxi trip makers. For taxi trip makers, it is observed (Table 3 and 4) that estimated value of alternative specific constant under traffic information scenario is higher than that of no-traffic-information scenario. This indicates that taxi trip makers' corridor choice behavior is comparatively more biased to choose less travel time and almost free-flow FO corridor under traffic information scenarios than no-trafficinformation scenario. This observation may be justified by the fact that trip makers generally hire taxies in the time of urgency or in instant-need. Under this circumstance, taxi trip- makers' corridor choice behavior is expected to be influenced greatly by traffic information in order to choose shorter travel time corridor for arriving at the destination comparatively early. Alternative specific constant term of a dis-utility model alone cannot determine the forecast of travel demand on alternative corridors. It is even largely influenced by saving in value of travel time under traffic information scenario. 
Table 1 - Procedure for Recalibration of Alternative Specific Constant Term using Combined RP and SP data sources: Traffic Information TI-I

\begin{tabular}{|c|c|c|c|c|c|c|}
\hline \multirow{3}{*}{ Attribute } & \multicolumn{3}{|c|}{ Private Car Trip makers } & \multicolumn{3}{|c|}{ Taxi Trip makers } \\
\hline & $\begin{array}{c}\text { RP } \\
\text { Model }\end{array}$ & $\begin{array}{c}\text { SP } \\
\text { Model }\end{array}$ & $\begin{array}{l}\text { Recalibration } \\
\text { of } A S C^{*}\end{array}$ & $\begin{array}{c}\text { RP } \\
\text { Model }\end{array}$ & $\begin{array}{c}\text { SP } \\
\text { Model }\end{array}$ & $\begin{array}{l}\text { Recalibration } \\
\text { of } A S C^{*}\end{array}$ \\
\hline & $\begin{array}{l}\text { Coeff. } \\
(t \text {-stat })\end{array}$ & $\begin{array}{l}\text { Coeff. } \\
(t \text {-stat })\end{array}$ & $\begin{array}{l}\text { Coeff. } \\
(t \text {-stat })\end{array}$ & $\begin{array}{l}\text { Coeff. } \\
(t \text {-stat })\end{array}$ & $\begin{array}{l}\text { Coeff. } \\
(t \text {-stat })\end{array}$ & $\begin{array}{l}\text { Coeff. } \\
(t \text {-stat })\end{array}$ \\
\hline \multicolumn{7}{|c|}{ Coefficient of Attributes fixed at SP Parameter estimates during recalibration of ASC** } \\
\hline $\begin{array}{l}\text { Travel Time } \\
\text { (TT) } \\
\text { Travel Cost } \\
\text { (TC) }\end{array}$ & $\begin{array}{c}-0.253 \\
(-11.81) \\
-0.325 \\
(-14.48)\end{array}$ & $\begin{array}{l}-0.210 \\
(-22.49) \\
-0.177 \\
(-20.44)\end{array}$ & $\begin{array}{c}-0.210 \\
\text { (fixed parameter) } \\
-0.177 \\
\text { (fixed parameter) }\end{array}$ & $\begin{array}{c}-0.201 \\
(-13.89) \\
-0.109 \\
(-12.03)\end{array}$ & $\begin{array}{c}-0.217 \\
(-21.51) \\
-0.216 \\
(-19.07)\end{array}$ & $\begin{array}{c}-0.217 \\
\text { (fixed parameter) } \\
-0.216 \\
\text { (fixed parameter) }\end{array}$ \\
\hline \multicolumn{7}{|c|}{ Parameter Allowed to be estimated freely during recalibration of ASC** } \\
\hline Asc. for $\mathrm{FO}^{\dagger}$ & $\begin{array}{l}-0.273 \\
(-2.23)\end{array}$ & $\begin{array}{c}1.456 \\
(11.97)\end{array}$ & $\begin{array}{c}-0.464 \\
(-5.974)\end{array}$ & $\begin{array}{c}-1.369 \\
(-11.13)\end{array}$ & $\begin{array}{c}1.464 \\
(9.873)\end{array}$ & $\begin{array}{c}-1.140 \\
(-12.40)\end{array}$ \\
\hline $\begin{array}{l}\text { No. of } \\
\text { Observations }\end{array}$ & 955 & 3651 & 955 & 1074 & 3857 & 1074 \\
\hline $\begin{array}{l}\text { Log likelihood } \\
\text { function }\end{array}$ & -401.13 & -1467.0 & -433.35 & -476.06 & -1270.35 & -438.88 \\
\hline$\rho^{2}$ & 0.3846 & 0.23376 & 0.3352 & 0.3412 & 0.27313 & 0.3926 \\
\hline
\end{tabular}

Table 2 - Procedure for Recalibration of Alternative Specific Constant Term using Combined RP and SP data sources: Traffic Information TI-II

\begin{tabular}{|c|c|c|c|c|c|c|}
\hline \multirow{3}{*}{ Attributes } & \multicolumn{3}{|c|}{ Private Car Trip makers } & \multicolumn{3}{|c|}{ Taxi Trip makers } \\
\hline & $\begin{array}{c}R P \\
\text { Model }\end{array}$ & $\begin{array}{c}\text { SP } \\
\text { Model }\end{array}$ & $\begin{array}{l}\text { Recalibration } \\
\text { of } A S C^{*}\end{array}$ & $\begin{array}{c}R P \\
\text { Model }\end{array}$ & $\begin{array}{c}S P \\
\text { Model }\end{array}$ & $\begin{array}{c}\text { Recalibration } \\
\text { of } A S C^{*}\end{array}$ \\
\hline & $\begin{array}{l}\text { Coeff. } \\
(t \text {-stat })\end{array}$ & $\begin{array}{l}\text { Coeff. } \\
(t \text {-stat })\end{array}$ & $\begin{array}{l}\text { Coeff. } \\
(t-\text { stat })\end{array}$ & $\begin{array}{l}\text { Coeff. } \\
(t \text {-stat })\end{array}$ & $\begin{array}{l}\text { Coeff. } \\
(t \text {-stat })\end{array}$ & $\begin{array}{l}\text { Coeff. } \\
(t \text {-stat })\end{array}$ \\
\hline \multicolumn{7}{|c|}{ Coefficient of Attributes fixed at SP Parameter estimates during recalibration of ASC** } \\
\hline Travel Time (TT) & $\begin{array}{l}-0.253 \\
(-11.81)\end{array}$ & $\begin{array}{c}-0.222 \\
(-27.39)\end{array}$ & $\begin{array}{c}-0.222 \\
\text { (fixed parameter) }\end{array}$ & $\begin{array}{c}-0.201 \\
(-13.89)\end{array}$ & $\begin{array}{l}-0.163 \\
(-20.52)\end{array}$ & $\begin{array}{c}-0.163 \\
\text { (fixed parameter) }\end{array}$ \\
\hline Travel Cost (TC) & $\begin{array}{c}-0.325 \\
(-14.48)\end{array}$ & $\begin{array}{c}-0.180 \\
(-20.90)\end{array}$ & $\begin{array}{c}-0.180 \\
\text { (fixed parameter) }\end{array}$ & $\begin{array}{c}-0.109 \\
(-12.03)\end{array}$ & $\begin{array}{c}-0.161 \\
(-12.64)\end{array}$ & $\begin{array}{c}-0.161 \\
\text { (fixed parameter) }\end{array}$ \\
\hline \multicolumn{7}{|c|}{ Coefficient s of levels of Attribute left out during recalibration of ASC** } \\
\hline Very High Variation & & $\begin{array}{c}-0.943 \\
(-10.56)\end{array}$ & Left Out & & $\begin{array}{l}-0.867 \\
(-7.08)\end{array}$ & Left Out \\
\hline High Variation & & $\begin{array}{l}-0.239 \\
(-4.04)\end{array}$ & Left Out & & $\begin{array}{l}-0.325 \\
(-2.68)\end{array}$ & Left Out \\
\hline Medium Variation & & $\begin{array}{l}0.149 \\
(3.31)\end{array}$ & Left Out & & $\begin{array}{l}0.384 \\
(7.43)\end{array}$ & Left Out \\
\hline \multicolumn{7}{|c|}{ Low Variation (Considered as base level during effects coding of variation levels of traffic information ) } \\
\hline \multicolumn{7}{|c|}{ Parameter Allowed to be estimated freely during recalibration of ASC** } \\
\hline Asc. for $\mathrm{FO}^{\dagger}$ & $\begin{array}{l}-0.273 \\
(-2.23)\end{array}$ & -- & $\begin{array}{l}-0.207 \\
(-2.51)\end{array}$ & $\begin{array}{c}-1.369 \\
(-11.13)\end{array}$ & $\begin{array}{l}0.769 \\
(3.21)\end{array}$ & $\begin{array}{c}-0.955 \\
(-11.53)\end{array}$ \\
\hline No. of Observations & 955 & 4643 & 955 & 1074 & 5035 & 1074 \\
\hline $\begin{array}{l}\text { Log likelihood } \\
\text { function }\end{array}$ & -401.13 & -1916.66 & -344.63 & -476.06 & -1449.31 & -461.13 \\
\hline$\rho^{2}$ & 0.3846 & 0.256 & 0.4384 & 0.3412 & 0.2005 & 0.3618 \\
\hline
\end{tabular}

In the present study, the composite utility (i.e. travel dis-utility) function of demand forecasting model of corridor choice under traffic information scenarios is constructed as given below.

Under Traffic Information TI-I:

Private car trip makers:

Taxi trip makers:

$$
V_{\text {ComUtility }(T I-I)}=-0.210 \times \text { travel time }-0.177 \times \text { direct travel cost }-0.464_{A S C(F O)}
$$

$$
V_{\text {Comutility }(T I-I)}=-0.217 \times \text { travel time }-0.216 \times \text { Taxi fare }-1.14_{A S C(F O)}
$$

Similarly, the composite utility function of demand forecasting for traffic flow on alternative corridors under traffic information TI-I is given below

Private car trip makers:

$$
\begin{gathered}
V_{\text {ComUtility }(T I-I I)}=-0.222 \times \text { travel time }-0.943 \times \text { VeryHighVariation }-0.239 \times \text { HighVariation }+0.149 \\
\times \text { MediumVariation }-0.180 \times \text { direct travel cost }-0.207_{A C S(F O)}
\end{gathered}
$$

Taxi trip makers:

$$
\begin{gathered}
V_{\text {ComUtility }(T I-I I)}=-0.163 \times \text { travel time }-0.867 \times \text { VeryHighVariation }-0.325 \times \text { HighVariation }+0.384 \\
\times \text { MediumVariation }-0.161 \times \text { taxi fare }-0.955_{A S C(F O)}
\end{gathered}
$$

\section{Conclusion}

In the present work, an attempt has been undertaken to demonstrate a procedure for developing demand forecasting model using revealed preference (i.e. RP) and stated preference (i.e. SP) data sources. Here, a composite utility function of combined RP and SP data sources is constructed by discarding the RP attribute estimates and the SP constant terms, but by retaining the SP attribute estimates and the alternative specific constant terms recalibrated from RP data, while keeping 
coefficients of attributes at SP estimates. The work is empirically demonstrated through a case study taken in Kolkata, where a single VMS board is assumed to display traffic information at a junction of two alternative and competitive traffic corridors connecting two catchment areas. The demand forecasting models are developed for two types of VMS based traffic information scenario, which differ in terms of content displayed on VMS board. The demand forecasting models are also developed for two types of mode user such as private car and taxi. In the procedure of constructing composite-utility function, if any attribute is found to be unique to RP data source then it would be allowed to be estimated freely. Similarly, if any attribute is found to be unique to SP data source, then out of necessity that will be left out of the estimation process. Overall the work demonstrates an experience of developing composite utility function of demand forecasting model using RP and SP data sources.

The scopes of the present work do not account for the validation study of actual performance of the composite utility (dis-utility) function under traffic information scenario. Therefore, as a part of the future scope of the current study, a validation study of the demand forecasting models may be undertaken; when the VMS system under study is actually implemented. It would also be interesting to compare the performance study of the composite dis-utility function with that of combined RP-SP models estimated by either of sequential approach or by simultaneous approach.

\section{Acknowledgements}

The authors acknowledge many valuable suggestions and advices received from John Rose, University of South Australia on various aspects of logit model estimation process. The authors also acknowledge the comments and suggestions of anonymous reviewers, which have helped to improve the paper substantially.

\section{References}

Adler, J. L., \& Blue, V. J. (1998). Toward the Design of Intelligent Traveler Information Systems. Transportation Research Part C: Emerging Technologies, 6(3), 157-172.

Basu. D., \& Maitra, B. (2007). Valuing Attributes of Enhanced Traffic Information: An Experience in Kolkata, Transport, 22(3), 164-173.

Basu, D., \& Maitra, B. (2010a). Stated preference approach for valuation of travel time displayed as traffic information on a VMS board. Journal of Urban Planning and Development, 136(3), 214-224.

Basu, D., \& Maitra, B. (2010b). Evaluation of VMS-based traffic information using multiclass dynamic traffic assignment model: An Experience in Kolkata. Journal of Urban Planning and Development, 136(1), 104-113.

Baofeng, S., Zhicai, J., Leleur, S., \& Wenjing, W. (2005). Research review of socio-economic impact evaluation for VMS project on freeway. Proceedings of the 8th International IEE Conference on Intelligent Transportation Systems, Viena, Austria.

Ben-Akiva, M. E., \& Morikawa, T. (1990). Estimation of switching models from revealed preferences and stated intentions. Transportation Research Part-A, 24 (6), 485-495.

Bierlaire, M., Themans, M., \& Axhausen, K. (2006). Analysis of driver's response to real-time information in Switzerland. European Transport, 34, 21-41.

Bonsall, P., Firmin, P., Anderson, M., Palmer, I., \& Balmforth, P. (1997). Validating the results of a route choice simulator. Transportation Research Part C: Emerging Technologies, 5(6), 371-387.

Bradley, M. A., \& Daly, A. J. (1994). Use of the logit scaling approach to test rank-order and fatigue effects in stated preference data. Transportation 21(2), 167-184.

Chatterjee, K., Hounsell, N. B., Firmin, P. E., \& Bonsall, P. W. (2002). Driver response to variable message sign information in London. Transportation Research Part C, 10, 149-169.

He, R., Ran, B., Choi, K., \& Kornhauser, A. L. (2003). Evaluation of dynamic pricing using multi-class dynamic network model. Journal of Transportation Engineering, 129, 617-624.

Hensher, D. A., Rose, A., \& Greene, W. H. (2005). Applied Choice Analysis: A Primer, Cambridge University Press, and NY.

Hensher, D. A. (1998). Establishing a fare elasticity regime for urban passenger transport. Journal of Transport Economics and Policy, 32(2), 221-246.

Hensher, D. A., \& Bradley, M. (1993). Using stated response choice data to enrich revealed preference discrete choice models. Marketing Letters, 4(2), 139-151.

Khattak, A., Polydoropoulou, A., \& Ben-Akiva, M. (1996). Modeling revealed and stated pre-trip travel response to advanced traveler information systems. Transportation Research Record, 1537, 46-54.

Lam, W. H. K., \& Chan, K. S. (2001). A model for assessing the effects of dynamic travel time information via variable message signs, Transportation, 28, 79-99.

Lim, Y., \& Lee, S. (1997). An Integrated Dynamic Traffic Assignment Model for Responsive Signal Control and Variable Message Sign. Journal of the Eastern Asia Society for Transportation Studies, 2(3), 753-763.

Louviere, J. J., Hensher, D. A., \& Swait, J. D. (2000). Stated Choice Methods, Analysis and Applications. Cambridge University Press, NY.

McDonald, M., \& Richards, A. (1996). Urban incident management using integrated control and information systems. Proceedings of the 8th International Conference on Road Traffic Monitoring and Control, London, April, IEE Conference Publication, 422, 188- 191.

McFadden, D. (1974). Conditional Logit Analysis of Qualitative Choice Behavior. In P. Zarembka (ed.), Frontiers in Econometrics (pp. 105142), New York: Academic Press.

Peeta, S., Ramos, J. L., \& Pasupathy, R. (2000). Content of variable message signs and on-line driver behavior. Transportation Research Record, 1725, 103-108.

Peeta, S., \& Ramos, J. L. (2006). Driver response to variable message signs-based traffic information. IEE Proceedings - Intelligent Transportation System, 153(1), 2-10.

Richards, A., McDonald, M., \& Lyons, G. D. (1996). Urban congestion reduction using variable message signs. Proceedings of the 24th PTRC European Transport Forum, Seminar E, Part 2, Brunel University, U.K.

Train, K. (2003). Discrete Choice Methods with Simulation. Cambridge: Cambridge University Press. 